Review Article

\title{
Effectiveness and Safety of Abrocitinib in Patients with Moderate-to-Severe Atopic Dermatitis: A Systematic Review and Meta-Analysis of Randomized Clinical Trials
}

\author{
Hammad Ali Fadlalmola $\left(\mathbb{D},{ }^{1}\right.$ Muayad Saud Albadrani, ${ }^{2}$ Amal Mohamed Elhusein, ${ }^{3,4}$ \\ Wahieba E. Mohamedsalih, ${ }^{1}$ Veerabhadra D. S. Swamy, ${ }^{3}$ and Daniel Mon Mamanao ${ }^{1}$ \\ ${ }^{1}$ Nursing College, Taibah University, Medina, Saudi Arabia \\ ${ }^{2}$ Medicine College, Taibah University, Medina, Saudi Arabia \\ ${ }^{3}$ College of Applied Medical Science, Bisha University, Bisha, Saudi Arabia \\ ${ }^{4}$ College of Nursing, Khartoum University, Khartoum, Sudan
}

Correspondence should be addressed to Hammad Ali Fadlalmola; hazzminno345@gmail.com

Received 10 April 2021; Revised 6 June 2021; Accepted 11 June 2021; Published 23 June 2021

Academic Editor: Giuseppe Stinco

Copyright (C) 2021 Hammad Ali Fadlalmola et al. This is an open access article distributed under the Creative Commons Attribution License, which permits unrestricted use, distribution, and reproduction in any medium, provided the original work is properly cited.

\begin{abstract}
Background. Atopic dermatitis (AD) is a complex, chronic, inflammatory skin disease characterized by pruritic, intense itching, and eczematous lesions affecting about $25 \%$ of children and $2 \%$ to $3 \%$ of adults worldwide. Abrocitinib is a selective inhibitor of Janus kinase-1 (JAK1) enzyme inhibiting the inflammatory process. Therefore, we aimed to assess the efficacy and safety of abrocitinib for moderate-to-severe AD. Methods. We systematically searched PubMed, Cochrane, Web of Science, Scopus, and EczemATrials till Feb 1, 2021, for reliable trials. The analysis was conducted using an inverse-variance method. The results were pooled as mean difference/event rate and 95\% confidence interval. Results. Abrocitinib $100 \mathrm{mg}$ and $200 \mathrm{mg}$ were associated with higher IGA response, EASI-50\% responders, EASI-75\% responders, EASI-90\% responders, number of participants with at least 4point improvements in NRS, and quality of life measured by DLQI and CDLQI than placebo. Also, $100 \mathrm{mg}$ and $200 \mathrm{mg}$ were associated with lower SCORAD index, \%BSA, PSAAD index, and POEM index than placebo. Abrocitinib $100 \mathrm{mg}$ and $200 \mathrm{mg}$ were not associated with adverse events such as upper respiratory tract infection, nasopharyngitis, dermatitis, atopic, any serious adverse events, and death. Conclusion. Abrocitinib in dose $100 \mathrm{mg}$ or $200 \mathrm{mg}$ is an effective, well-tolerated, and promising drug in treating patients with moderate-to-severe atopic dermatitis. However, the analysis favored the efficacy of abrocitinib $200 \mathrm{mg}$ over $100 \mathrm{mg}$, but side effects such as nausea and headache are likely to occur more with $200 \mathrm{mg}$.
\end{abstract}

\section{Introduction}

Atopic dermatitis $(\mathrm{AD})$ is a complex, chronic, inflammatory skin disease characterized by pruritic, intense itching, and eczematous lesions. It is the most prevalent inflammatory dermal diseases, affecting $3-10 \%$ of adults and $15-25 \%$ of children in the USA [1] and up to $25 \%$ of children and $2 \%$ to $3 \%$ of adults worldwide [2]. Therefore, $\mathrm{AD}$ is frequently repeated in the clinical practice [3]. The origin of $\mathrm{AD}$ is multifocal, including mainly encountered triggers due to skin barrier dysfunction that leads to enhanced skin irritability to nonspecific stimuli and epicutaneous sensitization
$[3,4]$. The primary risk factors for atopic dermatitis are the family history of eczema, allergies, hay fever or asthma, and the first year of life exposure to high levels of ambient nitrogen dioxide $\left(\mathrm{NO}_{2}\right)$ [5]. It is relapsing in nature, often with repeated flares, and may negatively impact the quality of life (QoL) for patients and their family members $[5,6]$.

There is no specific first-line medication for AD till now; however, some new medications are under investigation. The most frequent used medications for $\mathrm{AD}$ are symptomatic medications including emollients (moisturizers) used every day to prevent the skin from becoming dry, topical corticosteroids to reduce swelling and redness during 
flare-ups, antihistamines to reduce severe itching $[7,8]$. Hence, additional treatments are needed for patients with moderate-to-severe AD.

Recently, there were other family medications for treating some immune-related condition and AD: Janus kinase-1 inhibitor (JAK) inhibitors. JAK inhibitors belong to a family of medicines called DMARDs (disease-modifying antirheumatic drugs). FDA approves three JAK inhibitors: baricitinib, tofacitinib, and upadacitinib, for treating rheumatoid arthritis [9]. Pharmacologically, JAK inhibitors are small molecular synthetic compounds inhibiting the intracellular signal transduction of cytokine receptors [10]. Cytokines are mediators in numerous inflammatory skin disorder. These cytokines, especially interleukin I and II, are involved in the process of skin damage in $\mathrm{AD}$. JAK inhibitors work by modifying the immune system via inhibiting cytokine receptors by phosphorylation, decreasing the immune response, and improving $\mathrm{AD}$ symptoms [11]. JAK inhibitors are under clinical investigation for inflammatory skin diseases, specifically phase 3 trials for AD or psoriasis. As JAK inhibitors are tested in oral and topical formulations, they could become prevalent in dermal therapy [12].

Abrocitinib is a selective inhibitor of JAK1 enzyme [13]. JAK1 is a human tyrosine kinase protein important for signaling certain types of cytokines (type I and type II) and interacts with the common gamma chain $(\gamma c)$ of type I cytokine receptors to elicit signals from the interleukin-2 (IL-2) receptor family initiating responses to multiple major cytokine receptor families $[13,14]$.

As JAK-1 inhibitors are a new promising drug family for treating $\mathrm{AD}$ and due to the lack of evidence regarding the new drug called abrocitinib, although there are very few systematic reviews that talk about abrocitinib and atopic dermatitis, this systematic review examined several outcomes and different doses 100 and $200 \mathrm{mg}$ of treatment, resulting in a good evidence that may serve as a good reference to atopic dermatitis guidelines. In this systematic review and meta-analysis, we aimed to synthesize evidence regarding the efficacy and safety of abrocitinib $100 \mathrm{mg}$ and $200 \mathrm{mg}$ compared to placebo for patients suffering from AD.

\section{Methods}

2.1. Search Strategy and Data Collection. We searched five electronic databases, PubMed, Cochrane CENTRAL, Web of Science, Scopus, and Global Resource for EczemATrials (GREAT) (Centre of Evidence-Based Dermatology; http:// www.greatdatabase.org.uk), for all published clinical trials till Feb $1^{\text {st }}, 2021$. Also, we searched for any published results from ongoing studies on the ongoing trials registry of the US National Institutes of Health (http://www.clinicaltrials.gov). The search was conducted using the following search strategy: (Abrocitinib OR Janus kinase 1 inhibitor OR JAK1 inhibitor OR PF-04965842) AND (Atopic dermatitis OR Atopic Dermatitides OR Atopic Neurodermatitides OR Atopic Neurodermatitis OR Disseminated Neurodermatitides OR Disseminated Neurodermatitis OR Neurodermatitis Disseminata OR Atopic Eczema OR Infantile
Eczema OR flexural eczema OR prurigo Besnier OR allergic eczema OR Eczema Pruriginosum Allergicum).

Using Endnote software, we removed the duplicates; then, we screened all retrieved citations for eligibility through two steps: title and abstracts, then full text, and those matching our criteria were included in our study. We also screened the references of the included studies manually for additional relevant papers.

2.2. Selection Criteria. We included all randomized clinical trials (RCTs) that enrolled patients with moderate-to-severe atopic dermatitis and investigated abrocitinib's safety or efficacy in any dose compared to placebo. If more than one trial reported the same population, we used the most complete dataset of results. No restrictions for age, sex, site, or publication date were applied.

We excluded animal studies, observational studies, nonEnglish studies, nonavailable studies, thesis reviews, and if the abstract only is available.

2.3. Data Extraction. We extracted data related to the following: (1) summary of the included trials including: study time and sites, design and phase, protocol NCT number, total number of patients, inclusion criteria, study arms and number of patients in each, dose, route, and regimen of abrocitinib, duration of treatment, AD severity, study conclusion, (2) baseline characteristics of the enrolled population including: age, sex, race, disease duration, Investigator's Global Assessment (IGA) grade, Eczema Area and Severity Index (EASI) score, \% body surface area (BSA) affected, pruritus numeric rating scale (NRS) score, Scoring Atopic Dermatitis (SCORAD), Pruritus and Symptoms Assessment for Atopic Dermatitis (PSAAD), Patient Oriented Eczema Measure (POEM), Dermatology Life Quality Index (DLQI), Children's Dermatology Life Quality Index (CDLQI), previous medications for AD, (3) efficacy and safety outcomes, and (4) quality assessment domains.

2.4. Study Outcomes. We assessed the efficacy of abrocitinib in patients with $\mathrm{AD}$ through the following outcomes: IGA response, EASI 50\%, 75\%, 90\% responders, participants with at least four points improvements in NRS, SCORAD Index, \%BSA, PSAAD index, POEM index, quality of life by DLQI and CDQLI. Extracted safety measures included: death, serious adverse events, nausea, headache, dermatitis, atopic, nasopharyngitis, upper respiratory tract infection.

2.5. Quality Assessment. We assessed the quality of the included RCTs using Cochrane's risk of bias tool (version 1). The tool is found in chapter 8.5 of the Cochrane handbook of systematic reviews of interventions 5.1.0 [15]. The tool consists of the following domains: sequence generation (selection bias), allocation sequence concealment (selection bias), blinding of participants and personnel (performance bias), blinding of outcome assessors (detection bias), incomplete outcome data (attrition bias), selective outcome reporting (reporting bias), and other bias; author judgments 
fall into three categories: low, unclear, or high risk of bias for each domain.

We could not assess the risk of publication bias due to the small number of included studies, according to Egger's funnel-plot-based method [16].

2.6. Statistical Analysis. Continuous data were pooled as mean differences (MD) and 95\% confidence intervals (CI) using the inverse-variance method, while dichotomous data were pooled as risk ratio (RR) and 95\% CI using the Mantel-Haenszel method. We used the fixed-effect model when the pooled data are homogenous; otherwise, we used the random-effects model.

We used the Review Manager Software, version 5.3, to conduct the analysis. When the mean or standard deviation data are missing, we calculated their 95\% CI according to Altman's equation [17].

\section{Results}

3.1. Literature Search Results. Our search retrieved 158 citations after removing duplications, 140 records were excluded by title and abstract screening, and the remaining 18 were eligible for full-text screening. We finally included four trials in our study $[13,18-20]$. The flow of data collection and screening process are shown in (Figure 1).

3.2. Summary of the Included Studies. The included trials compared between different drug doses (10, 30, 100, $200 \mathrm{mg})$ and placebo with a total sample size of 1882 patients. All patients had moderate-to-severe $\mathrm{AD}$ and received the drug or placebo orally, once daily for nearly 12 weeks. Mean age of included patients ranged from 31 to 45 years with at least 20 years disease duration. Summary of the included trials and baseline characteristics of enrolled subjects is shown in Tables 1 and 2, respectively.

3.3. Quality Assessment. The risk of bias assessment revealed that the included studies were high quality according to the Cochrane risk of bias tool. All studies were at low risk of bias regarding selection, except one, detection attrition performance bias. One trial [19] was unclear the risk of selection bias. Regarding reporting bias, two trials were low risk $[18,20]$, one trial was unclear [19], and the remaining was high risk [13]. Three studies $[13,18,20]$ showed other sources of bias and the remaining was unclear [19]. Risk of bias graph and summary are shown in Figure 2.

\section{Outcomes}

4.1. IGA Response. Pooled analysis revealed that 100 and $200 \mathrm{mg}$ abrocitinib significantly increased IGA response more than placebo ( $\mathrm{RR}=3.03 ; 95 \%$ CI: $[2.14,4.30]$, $P<0.0001)$ (Figure 3) $(\mathrm{RR}=4.44 ; 95 \% \mathrm{CI}:[3.16,6.24]$, $P<0.0001$ ) (Supplementary Figure S1), respectively. Also, $200 \mathrm{mg}$ abrocitinib was associated with higher IGA response more than $100 \mathrm{mg}(\mathrm{RR}=1.47 ; 95 \%$ CI: $[1.26,1.72]$,

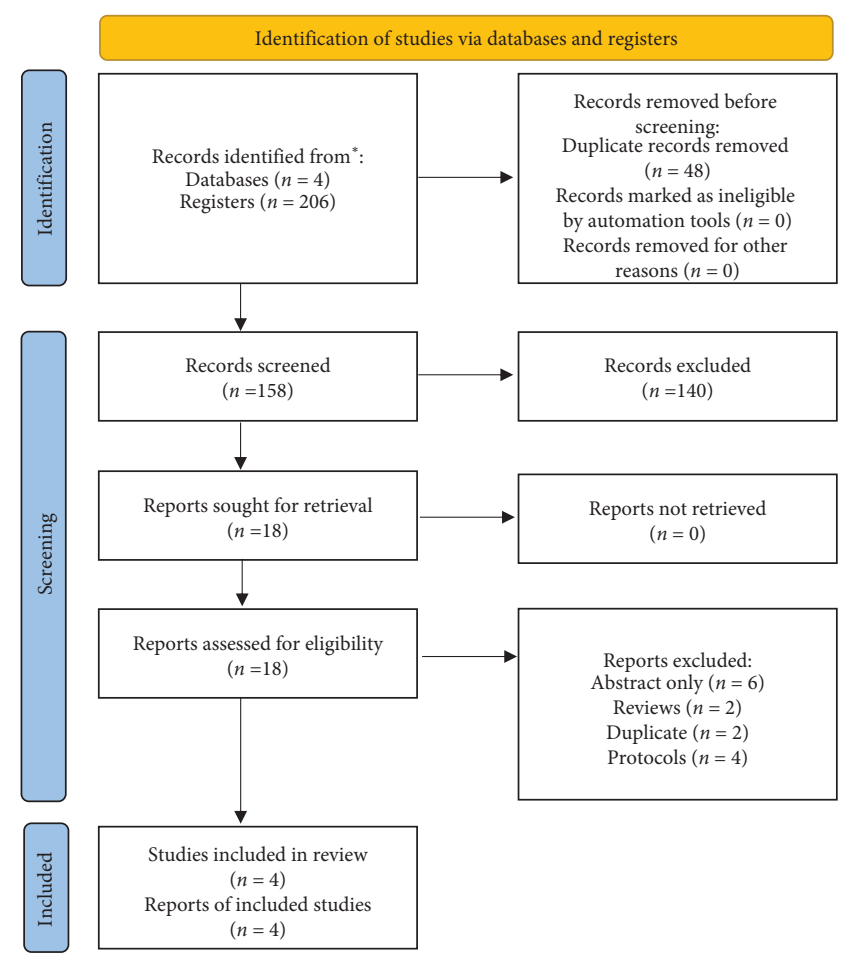

FIgURE 1: PRISMA flow diagram. It summarizes the results of searching databases and screening the obtained records.

$P<0.0001)$ (Supplementary Figure S2). Pooled results were homogenous $\left(I^{2}=0 \%, P=0.80\right)\left(I^{2}=0 \%, P=0.62\right)\left(I^{2}=0 \%\right.$, $P=0.47)$, respectively.

4.2. EASI-50 Responders. Pooled analysis revealed that 100 and $200 \mathrm{mg}$ abrocitinib significantly increased EASI-50 responders more than placebo ( $\mathrm{RR}=2.22 ; 95 \% \mathrm{CI}$ : $[1.38,3.58]$, $P=0.001) \quad(\mathrm{RR}=2.83 ; 95 \% \mathrm{CI}:[1.70,4.72], P<0.00001)$, respectively. Also, $200 \mathrm{mg}$ abrocitinib was associated with higher EASI-50 responders more than $100 \mathrm{mg}(\mathrm{RR}=1.23$; 95\% CI: $[1.15,1.32], P<0.00001)$. Pooled results were heterogenous in $100 \mathrm{mg}$ and $200 \mathrm{mg}$ vs. placebo $\left(I^{2}=84 \%\right.$, $P=0.0002)\left(I^{2}=88 \%, P<0.0001\right)$ and homogenous in 100 vs. $200 \mathrm{mg}\left(I^{2}=0 \%, P=0.44\right)$, respectively, and the heterogeneity was best resolved by excluding Pfizer (JADE compare trail) 2021 without effect on the significance.

4.3. EASI-75 Responders. Pooled analysis revealed that 100 and $200 \mathrm{mg}$ abrocitinib significantly increased EASI-75 responders more than placebo ( $\mathrm{RR}=2.74 ; 95 \% \mathrm{CI}$ : $[1.99,3.79]$, $P<0.00001)(\mathrm{RR}=4.04 ; 95 \% \mathrm{CI}:[2.55,6.42], P<0.00001)$, respectively. Also, $200 \mathrm{mg}$ abrocitinib was associated with higher EASI-75 responders more than $100 \mathrm{mg}(\mathrm{RR}=1.35$; 95\% CI: $[1.22,1.49], P<0.00001)$. Pooled results were heterogenous in $200 \mathrm{mg}$ vs. placebo $\left(I^{2}=65 \%, P=0.03\right)$ and homogenous in $100 \mathrm{mg}$ vs. placebo and $100 \mathrm{mg}$ vs. $200 \mathrm{mg}$ $\left(I^{2}=30 \%, P=0.23\right)\left(I^{2}=45 \%, P=0.14\right)$, respectively. The heterogeneity was best resolved by excluding Pfizer (JADE compare trail) 2021. 


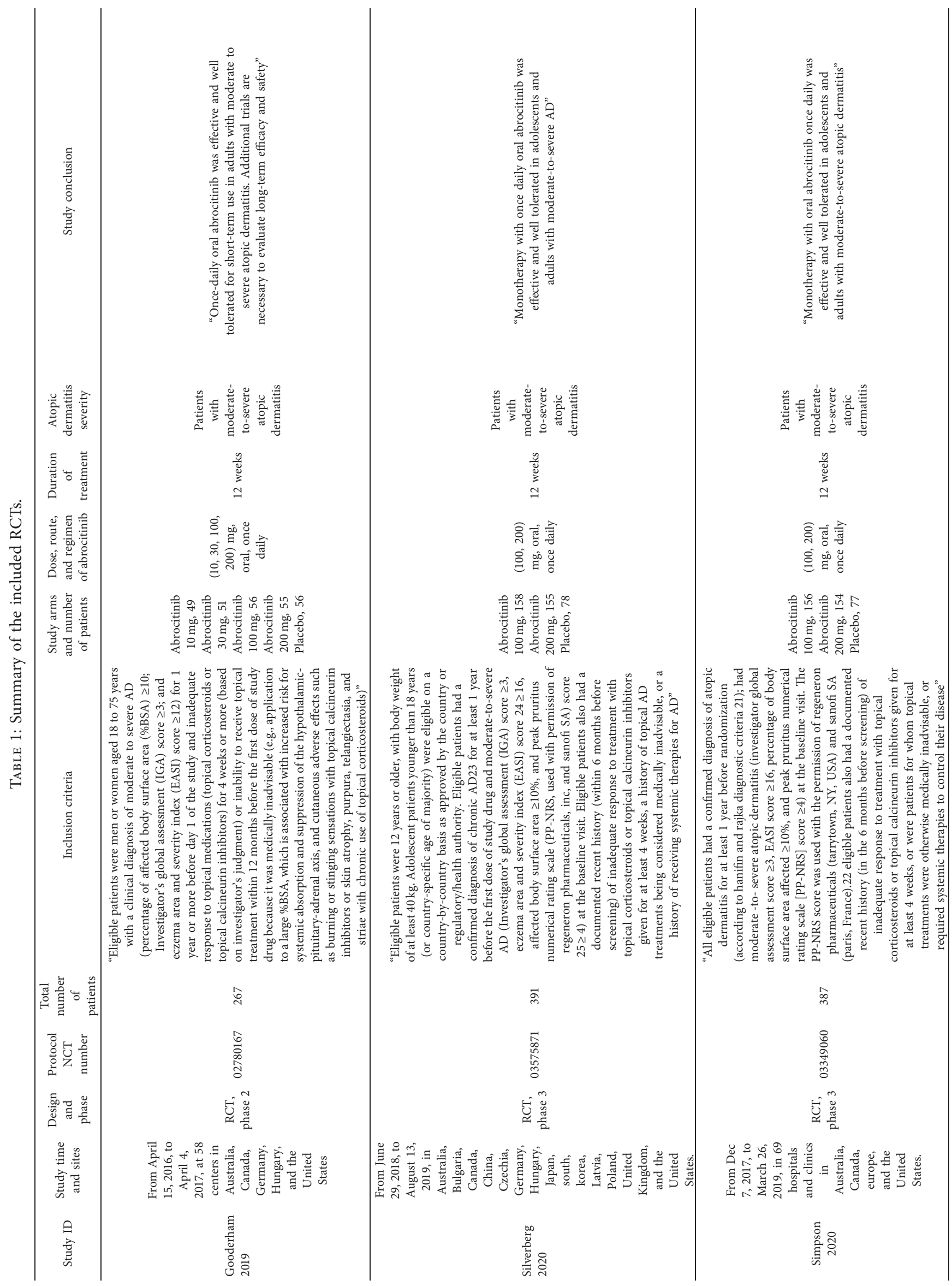




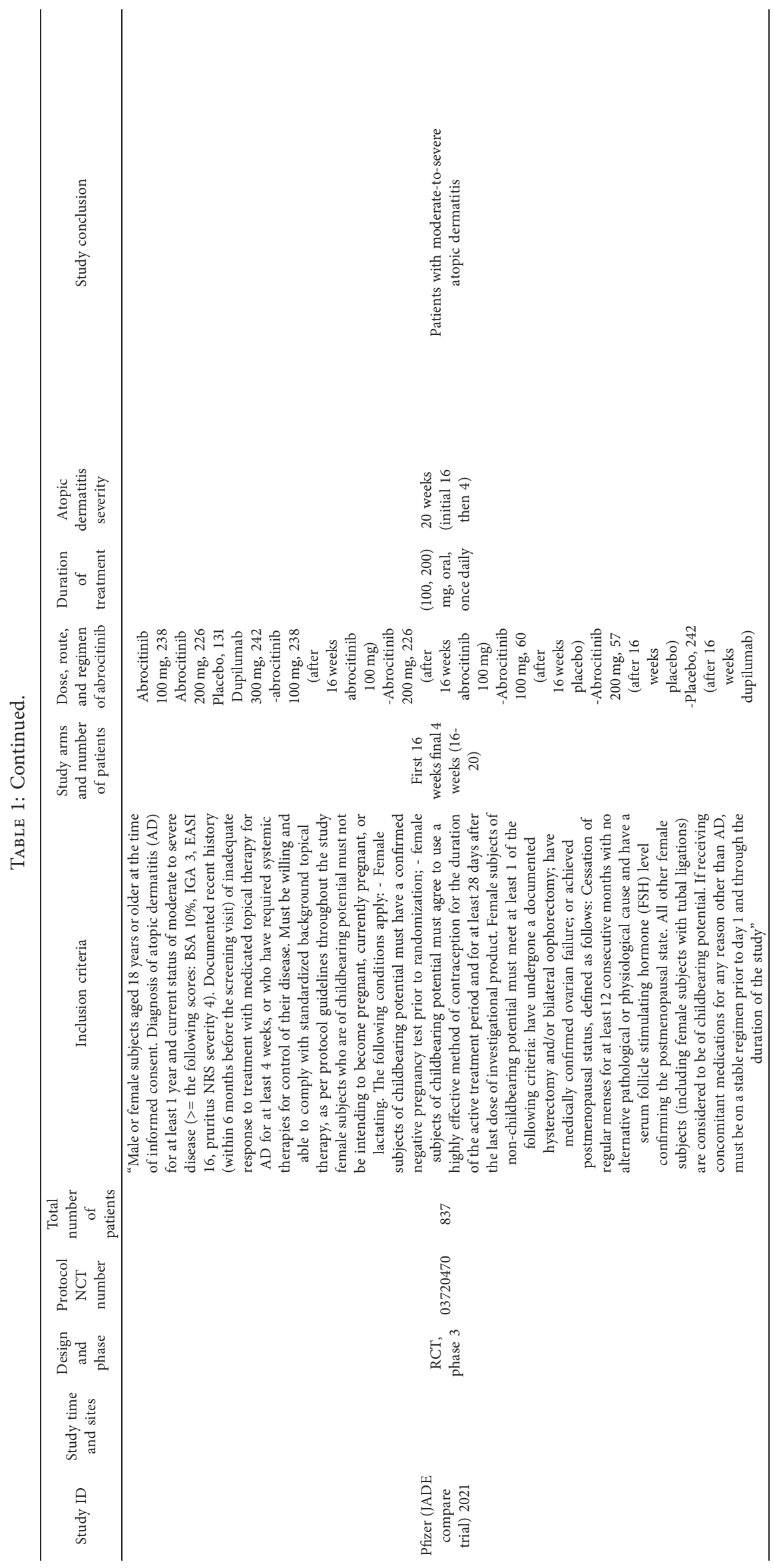




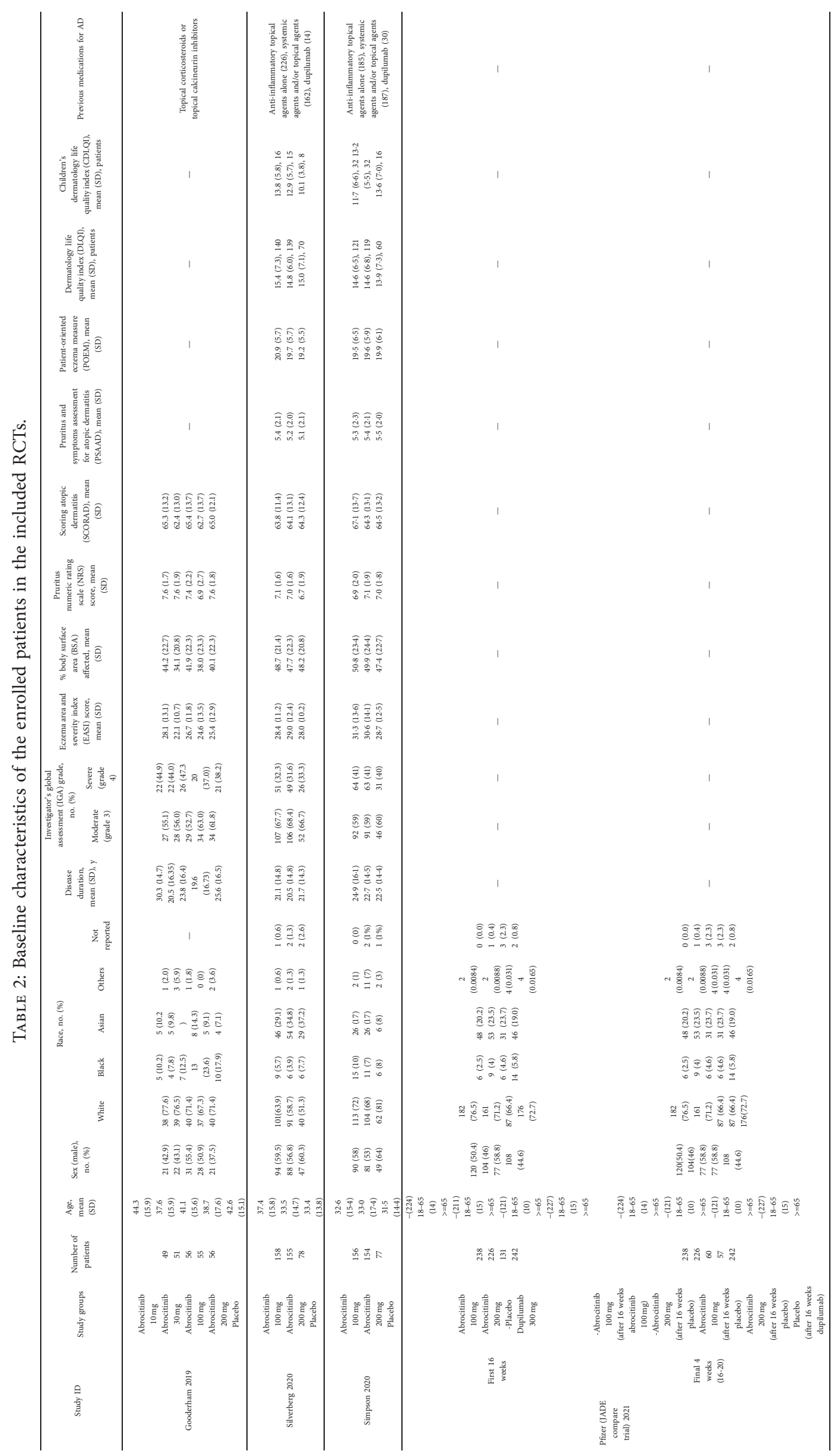




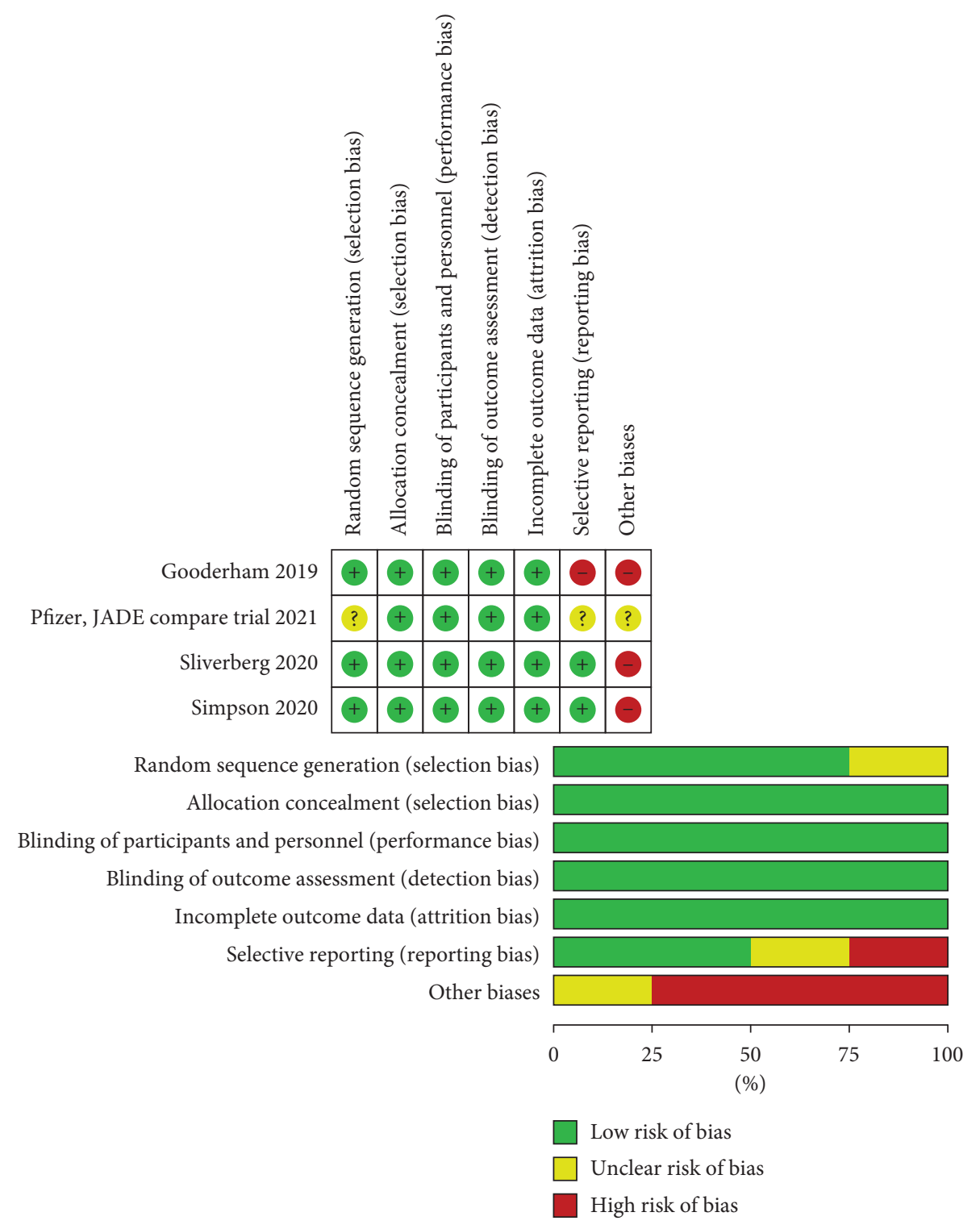

Figure 2: Risk of bias graph and summary.

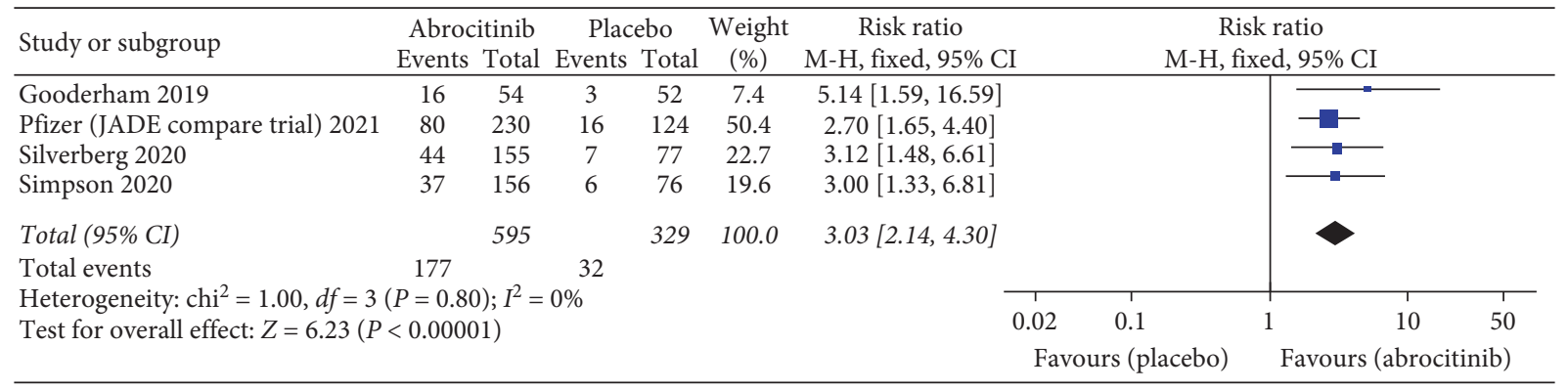

FIgURE 3: Forest plot of the IGA response (100 mg abrocitinib vs. placebo). 
4.4. EASI-90 Responders. Pooled analysis revealed that 100 and $200 \mathrm{mg}$ abrocitinib significantly increased EASI-90 responders more than placebo $(\mathrm{RR}=3.78 ; 95 \% \mathrm{CI}$ : $[2.53,5.65]$, $P<0.00001)(\mathrm{RR}=5.72$; 95\% CI: $[3.86,8.49], P<0.00001)$, respectively. Also, $200 \mathrm{mg}$ abrocitinib was associated with higher EASI-90 responders more than $100 \mathrm{mg}(\mathrm{RR}=1.51$; 95\% CI: $[1.29,1.78], P<0.00001)$. Pooled results were homogenous $\left(I^{2}=0 \%, P=0.75\right)\left(I^{2}=0 \%, P=0.57\right)\left(I^{2}=45 \%\right.$, $P=0.14)$, respectively.

EASI-50 responders, EASI-75 responders, and EASI-90 responders for $100 \mathrm{mg}$ abrocitinib vs. placebo are shown in Figure 4 while those for $200 \mathrm{mg}$ abrocitinib vs. placebo and $100 \mathrm{mg}$ vs. $200 \mathrm{mg}$ abrocitinib are shown in Supplementary Figures 3 and 4 .

4.5. Participants with at Least 4-Point Improvement in NRS. Pooled analysis revealed that 100 and $200 \mathrm{mg}$ abrocitinib significantly increased the number of participants with at least 4-point improvements in NRS more than placebo $(\mathrm{RR}=2.17 ; 95 \% \mathrm{CI}:[1.51,3.13], P<0.0001)(\mathrm{RR}=2.60 ; 95 \%$ CI: $[1.34,5.04], P=0.005$ ), respectively (Supplementary Figures 5 and 6), while there was no significant difference between $100 \mathrm{mg}$ and $200 \mathrm{mg}$ abrocitinib $(\mathrm{RR}=0.87$; 95\% CI: $[0.63,1.20], P=0.39$ ) (Supplementary Figure 7). Pooled results were heterogenous $\left(I^{2}=53 \%, P=0.09\right)\left(I^{2}=86 \%\right.$, $P<0.0001)\left(I^{2}=84 \%, P=0.0003\right)$, respectively, and the heterogeneity was best resolved by excluding Pfizer (JADE compare trail) 2021 in all three comparisons without effect on the significance except in $100 \mathrm{mg}$ vs. $200 \mathrm{mg}$ which became favoring $200 \mathrm{mg}$ in terms of increasing the number of patients with at least 4-point improvement in NRS (RR $=0.74 ; 95 \% \mathrm{CI}$ : [0.64, 0.86], $P=0.001)$.

4.6. SCORAD Index. Pooled analysis revealed that 100 and $200 \mathrm{mg}$ abrocitinib significantly reduced SCORAD index more than placebo $(\mathrm{MD}=(-13.33 ; 95 \% \mathrm{CI}:[-14.62,-12.05]$, $P<0.00001) \quad(\mathrm{MD}=-24.70 ; 95 \%$ CI: $[-25.98,-23.42]$, $P<0.00001$ ), respectively. Also, $200 \mathrm{mg}$ abrocitinib was associated with lower SCORAD index more than $100 \mathrm{mg}$ $(\mathrm{MD}=-10.83 ; 95 \% \mathrm{CI}:[-13.32,-8.34], P<0.00001)$. Pooled results were homogenous $\left(I^{2}=0 \%, P=0.98\right) \quad\left(I^{2}=0 \%\right.$, $P=0.32)\left(I^{2}=35 \%, P=0.0 .21\right)$, respectively.

4.7. \% BSA. Pooled analysis revealed that 100 and $200 \mathrm{mg}$ abrocitinib significantly reduced \% BSA more than placebo $(\mathrm{MD}=-10.92 ; 95 \%$ CI: $[-15.29,-6.55], \quad P<0.00001)$ $(\mathrm{MD}=-19.21 ; 95 \% \mathrm{CI}:[-23.56,-14.87], P<0.00001)$, respectively. Also, $200 \mathrm{mg}$ abrocitinib was associated with lower \% BSA more than $100 \mathrm{mg}(\mathrm{MD}=-8.33$; 95\% CI: $[-12.06,-4.60], P<0.0001)$. Pooled results were homogenous $\left(I^{2}=60 \%, P=0.12\right)\left(I^{2}=59 \%, P=0.12\right) \quad\left(I^{2}=0 \%\right.$, $P=0.98)$, respectively.

4.8. PSAAD Index. Pooled analysis revealed that 100 and $200 \mathrm{mg}$ abrocitinib significantly reduced PSAAD more than placebo $(\mathrm{MD}=-1.23 ; 95 \% \mathrm{CI}:[-1.54,-0.92]$, $P<0.00001) \quad(\mathrm{MD}=-2.08 ; \quad 95 \% \quad \mathrm{CI}: \quad[-2.39,-1.77]$,
$P<0.00001)$, respectively. Also, $200 \mathrm{mg}$ abrocitinib was associated with lower PSAAD more than $100 \mathrm{mg}$ $(\mathrm{MD}=-0.83 ; 95 \% \mathrm{CI}:[-1.09,-0.58], P<0.00001)$. Pooled results were homogenous $\left(I^{2}=0 \%, P=0.38\right)\left(I^{2}=0 \%\right.$, $P=0.86)\left(I^{2}=0 \%, P=0.48\right)$, respectively.

4.9. POEM Index. Pooled analysis revealed that 100 and $200 \mathrm{mg}$ abrocitinib significantly reduced \% BSA more than placebo $(\mathrm{MD}=-6.72 ; 95 \% \mathrm{CI}$ : $[-7.79,-5.65], P<0.00001)$ $(\mathrm{MD}=-7.33$; 95\% CI: $[-8.39,-6.26], P<0.00001)$, respectively, while there was no significant difference between $100 \mathrm{mg}$ and $200 \mathrm{mg}$ abrocitinib $(\mathrm{MD}=-0.73 ; 95 \% \mathrm{CI}$ : [ -2.19 , 0.73 ], $P=0.33)$. Pooled results were homogenous $\left(I^{2}=40 \%\right.$, $P=0.19) \quad\left(I^{2}=0 \%, \quad P=0.9\right) \quad\left(I^{2}=62 \%, \quad P=0.07\right)$, respectively.

SCORAD index, \% BSA, PSAAD index, and POEM index for $100 \mathrm{mg}$ abrocitinib vs. placebo are shown in Figure 5 while those for $200 \mathrm{mg}$ abrocitinib vs. placebo and $100 \mathrm{mg}$ vs. $200 \mathrm{mg}$ abrocitinib are shown in (Supplementary Figures 8 and 9).

4.10. DLQI. Pooled analysis revealed that 100 and $200 \mathrm{mg}$ abrocitinib significantly reduced PSAAD more than placebo $(\mathrm{MD}=-2.99 ; 95 \% \mathrm{CI}:[-3.88,-2.09], P<0.00001)(\mathrm{MD}=-$ 5.07; 95\% CI: $[-5.94,-4.20], P<0.00001)$, respectively. Also, $200 \mathrm{mg}$ abrocitinib was associated with lower PSAAD more than $100 \mathrm{mg} \quad(\mathrm{MD}=-2.06 ; 95 \%$ CI $\quad[-2.81,-1.30]$, $P<0.00001)$. Pooled results were homogenous $\left(I^{2}=32 \%\right.$, $P=0.23)\left(I^{2}=0 \%, P=0.6\right)\left(I^{2}=0 \%, P=0.67\right)$, respectively.

4.11. CDLQI. Pooled analysis revealed that 100 and $200 \mathrm{mg}$ abrocitinib significantly reduced \% BSA more than placebo $(\mathrm{MD}=-2.49 ; \quad 95 \% \quad \mathrm{CI}: \quad[-4.90, \quad-0.07], \quad P=0.04)$ $(\mathrm{MD}=-3.71 ; 95 \% \mathrm{CI}:[-6.13,-1.30], P=0.003)$, respectively, while there was no significant difference between $100 \mathrm{mg}$ and $200 \mathrm{mg}$ abrocitinib $(\mathrm{MD}=-1.23$; 95\% CI: [ -3.16 , 0.71 ], $P=0.21)$. Pooled results were homogenous $\left(I^{2}=0 \%\right.$, $P=0.95) \quad\left(I^{2}=0 \%, \quad P=0.62\right) \quad\left(I^{2}=0 \%, \quad P=0.49\right)$, respectively.

DLQI and CDLQI for $100 \mathrm{mg}$ abrocitinib vs. placebo, $200 \mathrm{mg}$ abrocitinib vs. placebo, and $100 \mathrm{mg}$ vs. $200 \mathrm{mg}$ abrocitinib are shown in Supplementary Figures 10-12, respectively.

4.12. Serious Adverse Events of Any Cause. Pooled analysis revealed no difference between 100 or $200 \mathrm{mg}$ abrocitinib and placebo $(\mathrm{RR}=0.81 ; 95 \% \mathrm{CI}$ : $[0.38,1.73], P=0.59)$ $(\mathrm{RR}=0.50 ; 95 \% \mathrm{CI}:[0.22,1.16], P=0.11)$, respectively. Also, there was no significant difference between $100 \mathrm{mg}$ and $200 \mathrm{mg}$ abrocitinib (RR $=1.59 ; 95 \%$ CI: [0.72, 3.53], $P=0.26)$. Pooled results were homogenous $\left(I^{2}=0 \%\right.$, $P=0.87) \quad\left(I^{2}=0 \%, \quad P=0.51\right) \quad\left(I^{2}=0 \%, \quad P=0.74\right)$, respectively.

4.13. Nausea. Pooled analysis revealed that 100 or $200 \mathrm{mg}$ abrocitinib was associated with higher incidence of nausea than placebo $(\mathrm{RR}=2.83 ; 95 \% \mathrm{CI}$ : $[1.26,6.35], P=0.01)$ 


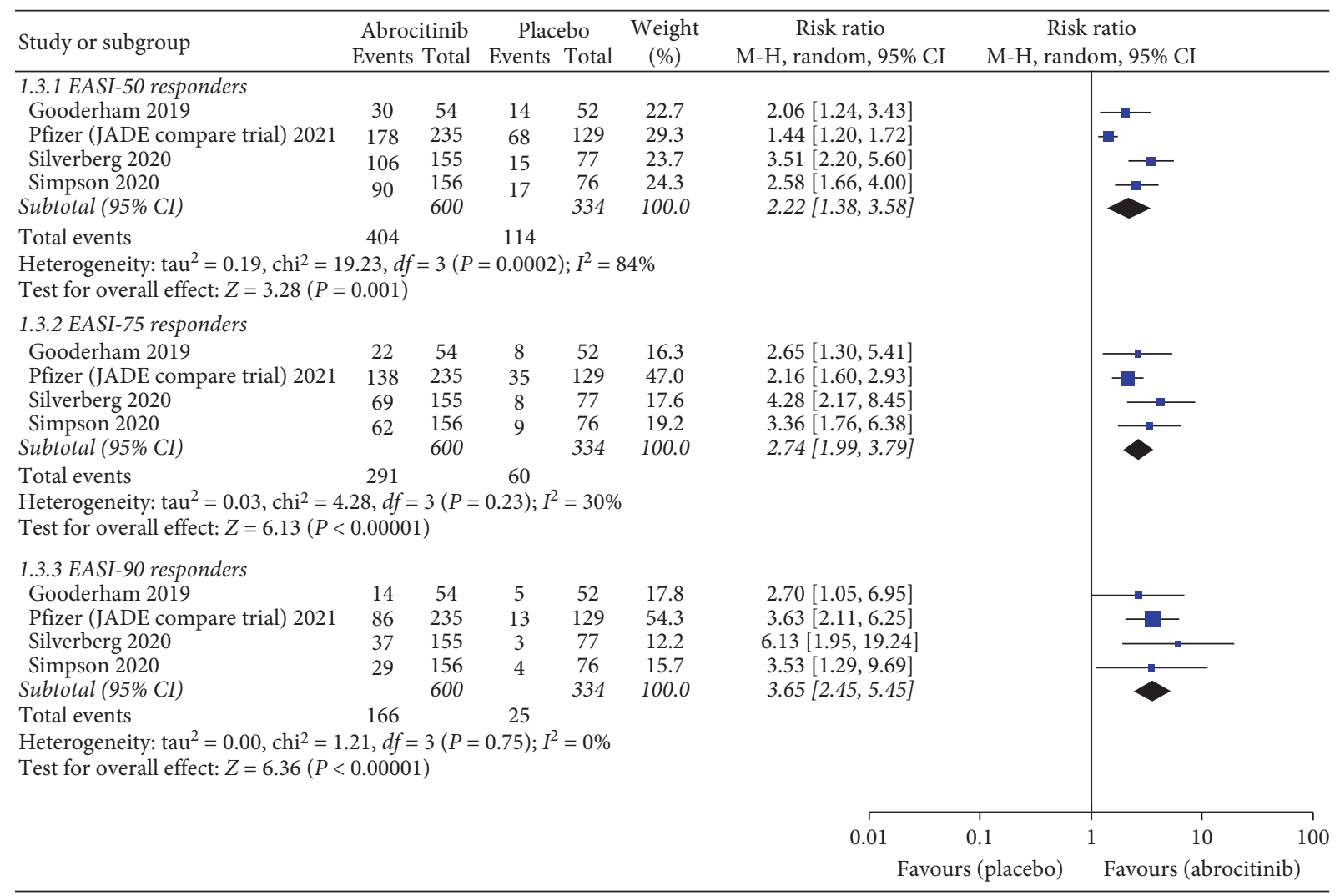

FIgURE 4: Forest plot of EASI-50, -75, and 90\% responders (100 mg abrocitinib vs. placebo).

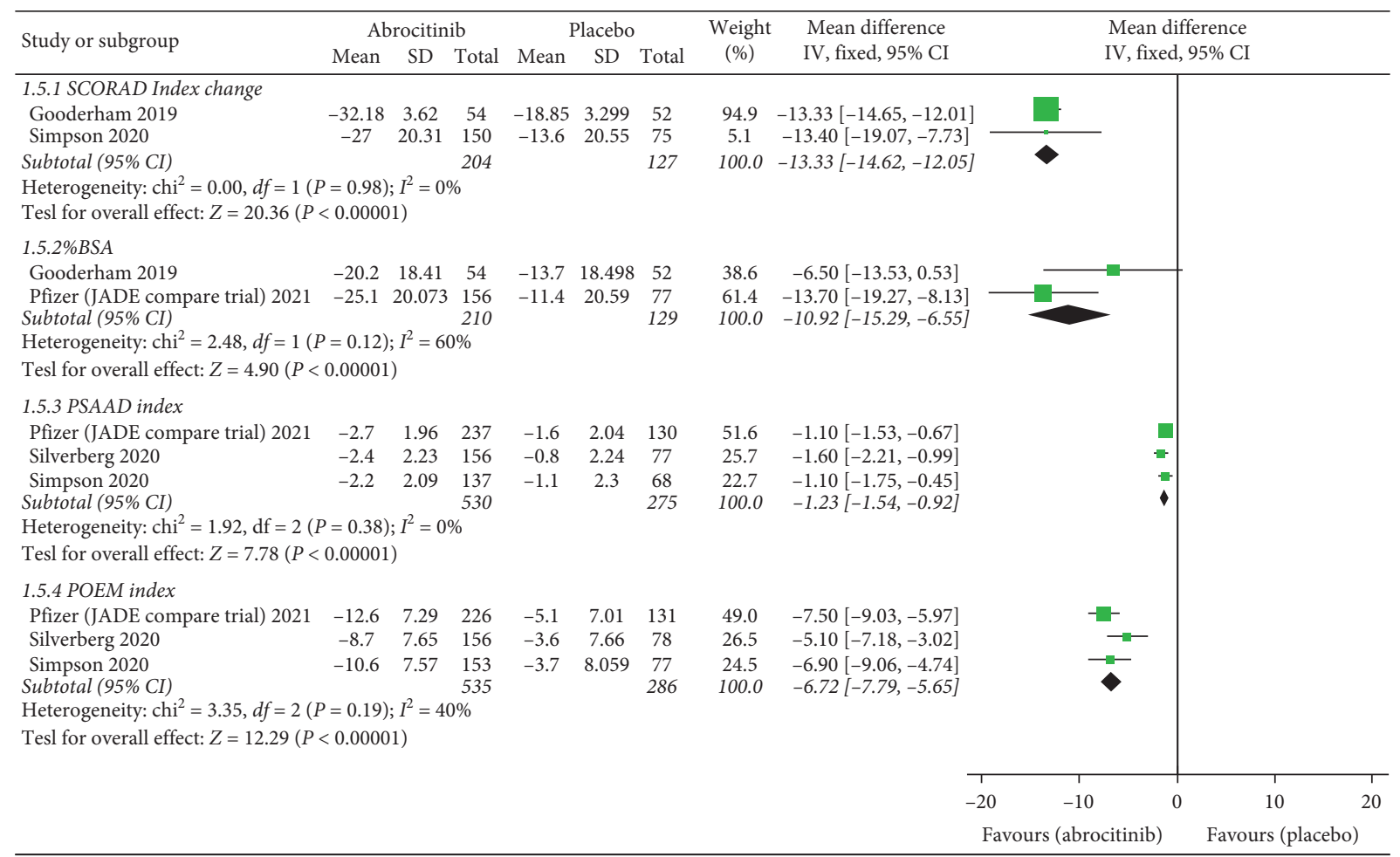

FIgUre 5: Forest plot of SCORAD index, \% BSA, PSAAD index, and POEM index (100 mg abrocitinib vs. placebo). 
$(\mathrm{RR}=6.98$; 95\% CI: [3.27, 14.92], $P<0.00001)$, respectively. Moreover, $200 \mathrm{mg}$ was associated with higher incidence of nausea than $100 \mathrm{mg}(\mathrm{RR}=0.42 ; 95 \% \mathrm{CI}$ : [0.29, 0.61], $P<0.00001)$. Pooled results were homogenous $\left(I^{2}=0 \%\right.$, $P=0.89) \quad\left(I^{2}=0 \%, \quad P=0.99\right) \quad\left(I^{2}=0 \%, \quad P=0.57\right)$, respectively.

4.14. Headache. Pooled analysis revealed no difference between 100 abrocitinib and placebo $(\mathrm{RR}=1.72 ; 95 \% \mathrm{CI}$ : [0.91, 3.27], $P=0.1$ ), while $200 \mathrm{mg}$ was associated with higher incidence of headache than placebo $(\mathrm{RR}=2.22 ; 95 \% \mathrm{CI}$ : [1.18, 4.16], $P=0.01$ ). Also, there was no significant difference between $100 \mathrm{mg}$ and $200 \mathrm{mg}$ abrocitinib $(\mathrm{RR}=0.76$; 95\% CI: [0.50, 1.16], $P=0.20)$. Pooled results were homogenous $\left(I^{2}=0 \%\right.$, $P=0.49)\left(I^{2}=0 \%, P=0.68\right)\left(I^{2}=0 \%, P=0.85\right.$

), respectively.

4.15. Dermatitis Atopic. Pooled analysis revealed no difference between 100 abrocitinib and placebo $(\mathrm{RR}=0.71 ; 95 \%$ CI: [0.47, 1.07], $P=0.1$, while $200 \mathrm{mg}$ was associated with lower incidence of dermatitis atopic than placebo $(R R=0.50$; 95\% CI: $[0.30,0.82], P=0.007)$. Also, there was no significant difference between $100 \mathrm{mg}$ and $200 \mathrm{mg}$ abrocitinib $(\mathrm{RR}=1.47 ; 95 \% \mathrm{CI}:[0.94,2.29], P=0.09)$. Pooled results were homogenous $\left(I^{2}=11 \%, P=0.34\right)\left(I^{2}=10 \%, P=0.34\right)$ $\left(I^{2}=50 \%, P=0.11\right)$, respectively.

4.16. Nasopharyngitis. Pooled analysis revealed no difference between 100 or $200 \mathrm{mg}$ abrocitinib and placebo $(\mathrm{RR}=1.52 ; 95 \% \mathrm{CI}:[0.96,2.41], P=0.08)(\mathrm{RR}=1.08 ; 95 \%$ CI: $[0.66,1.76], P=0.75)$, respectively. Also, there was no significant difference between $100 \mathrm{mg}$ and $200 \mathrm{mg}$ abrocitinib (RR $=1.40 ; 95 \% \mathrm{CI}$ : $[0.98,2.01], P=0.01)$. Pooled results were homogenous $\left(I^{2}=0 \%, P=0.81\right) \quad\left(I^{2}=0 \%\right.$, $P=0.94)\left(I^{2}=0 \%, P=0.85\right)$, respectively.

4.17. Upper Respiratory Tract Infection. Pooled analysis revealed no difference between 100 or $200 \mathrm{mg}$ abrocitinib and placebo $(\mathrm{RR}=1.20 ; 95 \% \mathrm{CI}$ : $[0.69,2.06], P=0.52)$ $(\mathrm{RR}=0.96 ; 95 \% \mathrm{CI}:[0.55,1.69], P=0.89)$, respectively. Also, there was no significant difference between $100 \mathrm{mg}$ and $200 \mathrm{mg}$ abrocitinib $(\mathrm{RR}=1.30 ; 95 \% \mathrm{CI}$ : [0.82, 2.06], $P=0.27)$. Pooled results were homogenous $\left(I^{2}=0 \%\right.$, $P=0.55) \quad\left(I^{2}=0 \%, \quad P=0.99\right) \quad\left(I^{2}=23 \%, \quad P=0.28\right)$, respectively.

Serious adverse events of any cause, nausea, headache, dermatitis, atopic, nasopharyngitis, and upper respiratory tract infection for $100 \mathrm{mg}$ abrocitinib vs. placebo are shown in Figure 6 while those for $200 \mathrm{mg}$ abrocitinib vs. placebo and $100 \mathrm{mg}$ vs. $200 \mathrm{mg}$ abrocitinib are shown in Supplementary Figures 13 and 14.

4.18. Death. Pooled analysis revealed no difference between 100 or $200 \mathrm{mg}$ abrocitinib and placebo $(\mathrm{RR}=0.858$; $95 \% \mathrm{CI}$ :
[0.136, 5.412], $P=0.87)(\mathrm{RR}=0.624 ; 95 \% \mathrm{CI}$ : [0.088, 4.403], $P=0.636$ ), respectively (Supplementary Figures 15 and 16). Also, there was no significant difference between $100 \mathrm{mg}$ and $200 \mathrm{mg}$ abrocitinib ( $\mathrm{RR}=1.407 ; 95 \% \mathrm{CI}$ : [0.223, 8.92], $P=0.716$ ) (Supplementary Figure 17). Pooled results were homogenous $\left(I^{2}=0 \%, P=0.97\right)\left(I^{2}=0 \%, P=0.99\right)\left(I^{2}=0 \%\right.$, $P=0.96)$, respectively.

\section{Discussion}

We found that $100 \mathrm{mg}$ and $200 \mathrm{mg}$ abrocitinib were not associated with adverse events such as upper respiratory tract infection, nasopharyngitis, dermatitis atopic, any serious adverse events, and death. However, some specific adverse events may occur with $100 \mathrm{mg}$ or $200 \mathrm{mg}$ as nausea and headache. The prevalence of nausea and headache was higher in $200 \mathrm{mg}$ than $100 \mathrm{mg}$. Regarding the efficacy, $100 \mathrm{mg}$ and $200 \mathrm{mg}$ were associated with higher IGA response, EASI-50\% responders, EASI-75\% responders, EASI$90 \%$ responders, number of participants with at least 4-point improvements in NRS, and quality of life measured by DLQI and CDLQI than placebo. Moreover, $100 \mathrm{mg}$ and $200 \mathrm{mg}$ were associated with lower SCORAD index, \%BSA, PSAAD index, and POEM index than placebo. Abrocitinib $200 \mathrm{mg}$ significantly increased IGA response, DLQI, EASI-50\% responders, EASI-75\% responders, EASI-90\% responders than $100 \mathrm{mg}$ while no difference regarding the number of participants with at least 4 points improvements in NRS, POEM index and CDLQI. Also, $200 \mathrm{mg}$ abrocitinib significantly decreased SCORAD index, \%BSA, PSAAD index than $100 \mathrm{mg}$.

JAK inhibitors are classed into several classes, as they can be used as immunomodulators as in case of DMARDS, tyrosine kinase inhibitors, and also inhibit cytokine activity modifying the immune system [21]. Tofacitinib is a treatment option for other autoimmune diseases and can also reduce pulmonary eosinophilia $[21,22]$. It was recently reported that patients with moderate-to-severe active ulcerative colitis treated with tofacitinib were more likely to have an improved clinical response than placebo [23]. Baricitinib has also proved efficacy in patients with active rheumatoid arthritis (RA) [21]. Upadacitinib is indicated for treating moderate-to-severe active RA in adults who have responded inadequately to or were intolerant to one or more DMARDs [24].

Regarding the outcome of Investigator's Global Assessment (IGA) scale response, it is a 5-point modified assessment tool which evaluates whether treatment, clinicians, and regulators meet the need for a valid, clinically meaningful measure or not [25]. It can be used for evaluating plaque psoriasis or atopic dermatitis severity in clinical trials. Langley et al.[25] concluded that the 5-point IGA scale is a valid measure of disease severity. We depend on IGA in our analysis, and it showed a higher efficacy favoring abrocitinib over placebo. All included studies $[13,18,20,26]$ in the analysis observed a significant improvement in the IGA scale in the abrocitinib 


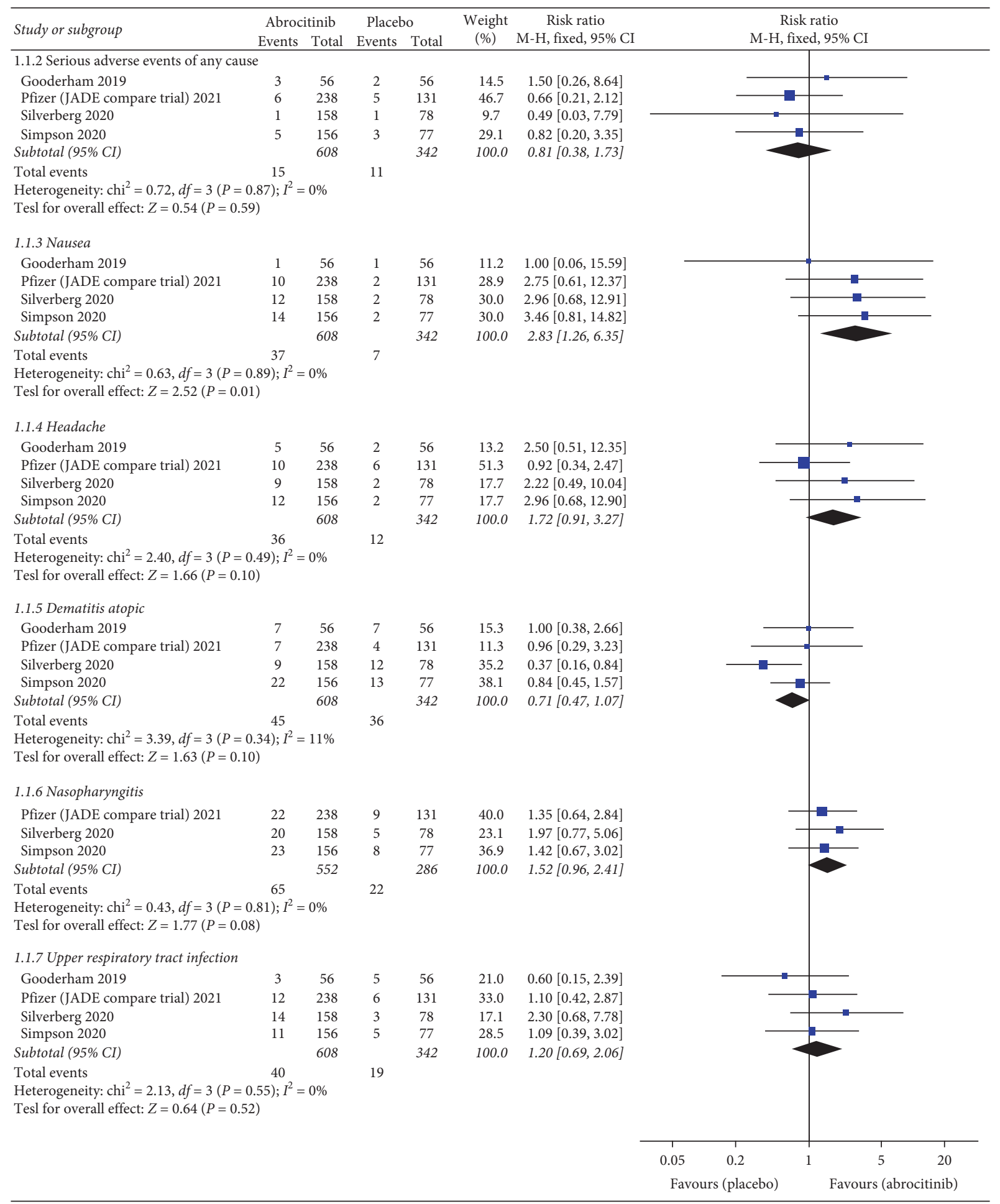

FIGURE 6: Forest plot of adverse events, serious adverse events of any cause, nausea, headache, dermatitis atopic, nasopharyngitis, and upper respiratory tract infection (100 $\mathrm{mg}$ abrocitinib vs. placebo).

group. However, a higher dose $(200 \mathrm{mg})$ showed a significant improvement rather than $100 \mathrm{mg}$.

Regarding the Eczema Area and Severity Index (EASI) score, it is an extensively validated scoring system that grades the physical signs of $\mathrm{AD}$ [27]. It is the core outcome for measuring the clinical signs of eczema in all trials.
Leshem et al. [28] provided the first guide for interpreting the EASI score. ESAI enables translation of the numerical output into an AD global severity state that should be more meaningful to providers and patients. EASI has demonstrated adequate feasibility, further supporting its use in clinical trials [28]. ESAI was reported by all included trials 
favoring abrocitinib over placebo. However, a higher dose $(200 \mathrm{mg})$ showed a significant improvement rather than $100 \mathrm{mg}$.

Regarding Pruritus Numerical Rating Scale (NRS) score, it is comprised of one item and represents the numbers 0 ("no itch") to 10 ("worst imaginable itch") [29]. Subjects are asked to rate the intensity of their itch using this scale with a simple format. It can be interpreted as follows: $\mathrm{NRS}=0$ indicates no pruritus, NRS $<3$ mild pruritus, NRS $>3<7$ moderate pruritus, NRS $\geq 7<9$ severe pruritus, and NRS $\geq 9$ very severe pruritus $[29,30]$. The NRS is a similar tool and has also been validated to measure pain [30]. NRS showed a significant result in our analysis favoring abrocitinib over placebo. There are other different scales for assessment we used in the analysis as Scoring Atopic Dermatitis (SCORAD) and Patient-Oriented Eczema Measure (POEM) [31]. SCORAD is a clinical tool used to assess the extent and severity of eczema [31]. Schram et al. [31] detected that SCORAD had fair responsiveness to atopic eczema. POEM is a validated, patient-derived assessment measure for monitoring atopic eczema severity [32].

It is the term of the quality of life (QoL) score outcome, and it was initially created by American psychologist John Flanagan in the 1970s. The QOL score was originally a 15-item that measured five conceptual domains of quality of life which are as follows: material and physical well-being, relationships with other people, social, community and civic activities, personal development and fulfilment, and recreation [33]. The QOLS is a valid instrument for measuring the quality of life across patient groups and cultures [33]. The quality of the included clinical trials meets a trusted level of evidence. We analyzed all available outcomes reported in the included trials with a considerable number of patients. All detected heterogeneity could be resolved. However, there are some limitations; we could not assess the publication bias due to the limited number of the included studies. The measured outcomes were assessed after limited duration of followup with no availability of long-term follow-up periods. We recommend future clinical trials with more sample sizes and strictly follow-up for longer durations.

\section{Conclusion}

Finally, we concluded that abrocitinib in dose $100 \mathrm{mg}$ or $200 \mathrm{mg}$ is an effective, well tolerated, and promising drug in treating patients with moderate-to-severe atopic dermatitis. However, the analysis favored the efficacy of abrocitinib $200 \mathrm{mg}$ over $100 \mathrm{mg}$, but side effects such as nausea and headache are likely to occur more with $200 \mathrm{mg}$.

\section{Data Availability}

All the data supporting the results of this study are available from the corresponding author upon request.

\section{Conflicts of Interest}

The authors declare that they have no conflicts of interest.

\section{Authors' Contributions}

HAF was the study leader and the design planner and conducted the analysis. HAF and MA screened the papers, extracted the data, and wrote the paper. VBS, AE, WE, and DM performed the following tasks independently: searching databases; screening records; data extraction. HAF, MA, and $\mathrm{AE}$ were responsible for editing, peer reviewing, and supervision.

\section{Supplementary Materials}

This file includes 17 figures in the form of forest plots of all measured outcomes with the legend of each figure, IGA response, EASI-50 responders, EASI-75 responders, EASI90 responders, participants with at least 4-point improvement in NRS, SCORAD index, \% BSA, PSAAD index, POEM index, DLQI, CDLQI, nausea, headache, dermatitis atopic, nasopharyngitis, and upper respiratory tract infection. (Supplementary Materials)

\section{References}

[1] H. He and E. Guttman-Yassky, "JAK inhibitors for atopic dermatitis: an update," American Journal of Clinical Dermatology, vol. 20, no. 2, pp. 181-192, 2019.

[2] L. F. Eichenfield, W. L. Tom, S. L. Chamlin et al., "Guidelines of care for the management of atopic dermatitis," Journal of the American Academy of Dermatology, vol. 70, no. 2, pp. 338-351, 2014.

[3] N. Katoh, Y. Ohya, M. Ikeda et al., "Japanese guidelines for atopic dermatitis 2020," Allergology International, vol. 69, no. 3, pp. 356-369, 2020.

[4] J. Wang and H. A. Sampson, "Atopic dermatitis and food hypersensitivity," Pediatric Allergy: Principles and Practice, vol. 12, pp. 414-419, 2016.

[5] S. Deng, D. Huang, W. Wang, H. Yan, S. Li, and H. Xiang, "Associations of gestational and the first year of life exposure to ambient air pollution with childhood eczema in Hubei, China," Environmental Science and Pollution Research, vol. 26, no. 23, pp. 23842-23849, 2019.

[6] D. Norbäck, C. Lu, Y. Zhang et al., "Sources of indoor particulate matter (PM) and outdoor air pollution in China in relation to asthma, wheeze, rhinitis and eczema among preschool children: synergistic effects between antibiotics use and PM10 and second hand smoke," Environment International, vol. 125, pp. 252-260, 2019.

[7] S. R. Janmohamed, A. P. Oranje, A. C. Devillers et al., "The proactive wet-wrap method with diluted corticosteroids versus emollients in children with atopic dermatitis: a prospective, randomized, double-blind, placebo-controlled trial," Journal of the American Academy of Dermatology, vol. 70, no. 6, pp. 1076-1082, 2014.

[8] L. Caminiti, "Allergen immunotherapy in atopic dermatitis: light and shadow in children," Pediatric Allergy and Immunology, vol. 31, no. S26, pp. 46-48, 2020.

[9] F. Wang, L. Sun, S. Wang et al., "Efficacy and safety of tofacitinib, baricitinib, and upadacitinib for rheumatoid arthritis: a systematic review and meta-analysis," Mayo Clinic Proceedings, vol. 95, no. 7, pp. 1404-1419, 2020.

[10] M. Gadina, N. Gazaniga, L. Vian, and Y. Furumoto, "Small molecules to the rescue: inhibition of cytokine signaling in 
immune-mediated diseases," Journal of Autoimmunity, vol. 85, pp. 20-31, 2017.

[11] C. M. Lin, F. A. Cooles, and J. D. Isaacs, "Basic mechanisms of JAK inhibition," Mediterranean Journal of Rheumatology, vol. 31, no. 1, pp. 100-104, 2020.

[12] F. Solimani, F. J. Hilke, and K. Ghoreschi, "Pharmakologie der Januskinaseinhibitoren," DerHautarzt, vol. 70, no. 12, pp. 934-941, 2019.

[13] M. J. Gooderham, S. B. Forman, R. Bissonnette et al., "Efficacy and safety of oral Janus kinase 1 inhibitor abrocitinib for patients with atopic dermatitis," JAMA Dermatology, vol. 155, no. 12, p. 1371, 2019.

[14] M. Gadina, D. Hilton, J. A. Johnston et al., "Signaling by Type I and II cytokine receptors: ten years after," Current Opinion in Immunology, vol. 13, no. 3, pp. 363-373, 2001.

[15] C. Reviews, C. Reviews, and T. C. Library: Cochrane handbook for systematic reviews of interventions handbook for systematic reviews of interventions chapter 1 : Introduction under construction please do not use key points.

[16] M. Egger, G. D. Smith, M. Schneider, and C. Minder, "Bias in meta-analysis detected by a simple, graphical test," Bmj, vol. 315 , no. 7109 , pp. 629-634, 1997.

[17] D. G. Altman and J. M. Bland, "Standard deviations and standard errors," BMJ, vol. 331, no. 7521, 903 pages, 2005.

[18] J. I. Silverberg, E. L. Simpson, J. P. Thyssen et al., "Efficacy and safety of abrocitinib in patients with moderate-to-severe atopic dermatitis," JAMA Dermatology, vol. 156, no. 8, p. 863, 2020.

[19] Pfizer, Study Evaluating Efficacy and Safety of PF-04965842 and Dupilumab in Adult Subjects with Moderate to Severe Atopic Dermatitis on Background Topical Therapy (JADE Compare) https://clinicaltrials.gov/ct2/show/NCT03720470, 2021.

[20] E. L. Simpson, R. Sinclair, S. Forman et al., "Efficacy and safety of abrocitinib in adults and adolescents with moderate-tosevere atopic dermatitis (JADE MONO-1): a multicentre, double-blind, randomised, placebo-controlled, phase 3 trial," The Lancet, vol. 396, no. 10246, pp. 255-266, 2020.

[21] Y. Furumoto and M. Gadina, "The arrival of jak inhibitors: advancing the treatment of immune and hematologic disorders," BioDrugs, vol. 27, no. 5, pp. 431-438, 2013.

[22] E. Kudlacz, M. Conklyn, C. Andresen, C. Whitney-Pickett, and P. Changelian, "The JAK-3 inhibitor CP-690550 is a potent anti-inflammatory agent in a murine model of pulmonary eosinophilia," European Journal of Pharmacology, vol. 582, no. 1-3, pp. 154-161, 2008.

[23] F. Balzola, G. Cullen, G. T. Ho, R. K. Russell, and J. Wehkamp, "Tofacitinib, an oral Janus kinase inhibitor," The New England Journal of Medicine, vol. 13, no. 2, pp. 70-71, 2012.

[24] NPS MedicineWise, "New drug: Upadacitinib for rheumatoid arthritis," Australian Prescriber, vol. 43, no. 5, pp. 178-179, 2020.

[25] R. G. B. Langley, S. R. Feldman, J. Nyirady, P. Van De Kerkhof, and C. Papavassilis, "The 5-point Investigator's Global Assessment (IGA) Scale: a modified tool for evaluating plaque psoriasis severity in clinical trials," Journal of Dermatological Treatment, vol. 26, no. 1, pp. 23-31, 2015.

[26] Study evaluating efficacy and safety of PF-04965842 and dupilumab in adult subjects with moderate to severe atopic dermatitis on background topical therapy full text viewClinicalTrials.Gov.

[27] J. Schmitt, P. I. Spuls, K. S. Thomas et al., "The Harmonising Outcome Measures for Eczema (HOME) statement to assess clinical signs of atopic eczema in trials," Journal of Allergy and Clinical Immunology, vol. 134, no. 4, pp. 800-807, 2014.

[28] Y. A. Leshem, T. Hajar, J. M. Hanifin, and E. L. Simpson, "What the Eczema Area and Severity Index score tells us about the severity of atopic dermatitis: an interpretability study," British Journal of Dermatology, vol. 172, no. 5, pp. 1353-1357, 2015.

[29] E. Verweyen, S. Ständer, K. Kreitz et al., "Validation of a comprehensive set of pruritus assessment instruments: the chronic pruritus tools questionnaire PRURITOOLS," Acta Dermato Venereologica, vol. 99, no. 7, pp. 657-663, 2019.

[30] N. Phan, C. Blome, F. Fritz et al., "Assessment of pruritus intensity: prospective study on validity and reliability of the visual analogue scale, numerical rating scale and verbal rating scale in 471 patients with chronic pruritus," Acta Dermato Venereologica, vol. 92, no. 5, pp. 502-507, 2012.

[31] M. E. Schram, P. I. Spuls, M. M. G. Leeflang et al., "EASI, (objective) SCORAD and POEM for atopic eczema: responsiveness and minimal clinically important difference," Allergy, vol. 67, no. 1, pp. 99-106, 2012.

[32] C. R. Charman, A. J. Venn, J. C. Ravenscroft, and H. C. Williams, "Translating Patient-Oriented Eczema Measure (POEM) scores into clinical practice by suggesting severity strata derived using anchor-based methods," British Journal of Dermatology, vol. 169, no. 6, pp. 1326-1332, 2013.

[33] C. S. Burckhardt and K. L. Anderson, "The quality of life scale (QOLS): reliability, validity, and utilization," Health and Quality of Life Outcomes, vol. 1, no. 1, p. 60, 2003. 